\title{
La teoría vygotskyana de los afectos ante el capitalismo emocional en la escuela
}

\section{The Vygotskian theory of affects in the face of emotional capitalism at school}

\author{
Alfonso André Bonhomme Manriquez ${ }^{1}$ \\ ${ }^{1}$ Psicólogo Educacional. Doctorando en Psicología en la Universidad Alberto Hurtado, \\ Santiago, Chile. E-mail: abonhomme@uahurtado.cl

\begin{abstract}
El autor agradece al Programa Doctoral de la Universidad Alberto Hurtado el financiamiento mediante una beca durante el primer año de sus estudios doctorales, y a la Agencia Nacional de Investigación y Desarrollo (ANID), del Gobierno de Chile, la beca de arancel y manutención para financiar actualmente sus estudios.

Facultad de Psicología, Universidad Alberto Hurtado.

Santiago de Chile.
\end{abstract}

\section{Resumen}

En la actualidad han cobrado relevancia los discursos sobre la importancia de atender la dimensión emocional en la escuela. Sin embargo, la conceptualización de las emociones en la escuela no le permite a esta desempeñar su rol transformador, confundiendo el papel de docentes y establecimientos y cayendo en determinismos que conllevan a la estigmatización del estudiante y/o su entorno. El presente artículo tiene como objetivo, por un lado, problematizar sobre las conceptualizaciones vigentes acerca de las emociones y su desarrollo, tomando en cuenta una investigación previa. Luego, se propone la revisión teórica de los principales conceptos de L.S. Vygotsky para entender las emociones y su desarrollo. La tesis principal es que las emociones se desarrollan siguiendo la misma lógica que Vygotsky define para el resto de los procesos psíquicos superiores, y que urge una teoría de las emociones que permita dar respuesta a las necesidades y problemas que tiene hoy la escuela.

Palabras clave: emociones, afectos, desarrollo, educación, Vygotsky

\begin{abstract}
Today, emotional capitalism permeates much of the space in which subjects perform in society. A good example of this, are the conceptions about emotions in schools. The dominant discourses of emotions become devices oriented to the reproduction of a social order, in which psychology assigns itself an important role, becoming itself a fundamental tool in schools for the containment of emotions that are ultimately the product of social inequality and stigmatization for which States should take responsibility.

In addition, studies on emotions conducted from an educational and a psychological perspective have also proliferated.

These studies on the affective dimension in the educational sphere in general share three core aspects: I) the predominance of the cognitive paradigm and the emotional intelligence model, which regard emotions as skills and/or competences at the service of adaptability; II) the incorporation of mental health goals at school in order to legitimize the inclusion of emotions in the educational domain; and III) the reconfiguration of teacher and
\end{abstract}


student roles at school, incorporating attachment theories to our understanding of the teacher-student relationship, ascribing a therapeutic/repairing function to it.

So far, psychology has provided a dualistic and abstract theory of emotions consistent with the aforementioned discourses. However, it is possible to think of theories that allow schools to fulfil their transforming social role.

The objective of this article is, on the one hand, to problematize the current conceptualizations about emotions and their development, taking into account previous research. On the other hand, the theoretical revision of the main concepts of L.S. Vygotsky is proposed in order to understand emotions and their development.

The main thesis is that emotions are developed following the same logic that Vygotsky defines for the rest of the superior psychic processes, and that there is an urgent need for a theory of emotions that allows us to respond to the needs and problems that the school has today.

The present article proposes the concept of emotions that emerges from Vygotsky's work as an alternative to existing theories and that fulfils the above-mentioned characteristics. In order to do so, it is necessary to elaborate a (re)construction of the concept of emotions present in the work of this author, in coherence with his solid theory of psychological processes.

In the first place, the vygotskyana perspective will be analyzed as a monist perspective regarding intellect and emotion. Vygotsky is critical of the Cartesian tradition, which gives rise to centripetal theories (peripheral and mechanistic) and centrifugal theories (centralist and spiritualist) of emotions. These perspectives presuppose a dualistic vision between body and mind. The relevance of language as a fundamental tool in human historical-cultural ontogenetic development and the configuration of higher psychological systems will then be discussed. The concepts of psychological systems and transformation will also be deepened in order to understand the development of diverse psychological processes, including emotions.

Finally, the concept of emotions that is at the base of educational theories and proposals does matter. Psychology is largely responsible for the confusing implications analyzed that impede the adequate development of the transforming potential of the school. Vygotsky's theory seems to give better lights and open the way to effective and responsible strategies, ethically and politically speaking, by incorporating the emotional dimension into the educational field.

Keywords: emotions, affects, development, education, Vygotsky

\section{Introducción}

Las emociones impregnan actualmente gran parte de la vida social. En los últimos años ha emergido una creciente preocupación por entender cómo las emociones cumplen un rol importante en distintas áreas en las que el ser humano rinde en sociedad. Esto se puede ver en cómo han proliferado en Chile nuevas líneas de investigación en lo que se denomina el desarrollo socioemocional, principalmente orientado al ámbito educacional (Álamos, Alcalay, Berger y Milicic, 2013; Álamos, Marchant y Milicic, 2015; Berger y Toro, 2012; Carrasco, Figueroa, Guzman-Gonzalez, Trabbuco y Vilca, 2016; Casassus, 2007; Céspedes y Silva, 2013; otros/as)

La relevancia de atender las emociones y el bienestar emocional en las aulas y escuelas se ve reflejada en las modificaciones que ha sufrido el Sistema de Medición de la Calidad de la Educación (SIMCE) que se aplica cada cierto tiempo a todos los establecimientos escolares con el fin de evaluar los resultados de aprendizaje a nivel nacional (Agencia de Calidad de la Educación, 2018). En el año 2014 se incorporó un cuestionario que mide indicadores de desarrollo personal y social en el que se encuentran los factores de autoestima académica, motivación escolar y convivencia 
escolar, entre otros (Agencia de Calidad de la Educación, 2014). Por esto, se puede apreciar la importancia que han ido adquiriendo los aspectos no académicos en la educación, que se enfocan principalmente en la relación de los estudiantes consigo mismos, con sus pares y con los profesores.

Organizaciones internacionales tales como el Banco Mundial y la Organización para Cooperación y el Desarrollo Económico (OCDE) propulsan a los países a desarrollar propuestas de ley en cuanto a educación emocional. Países vecinos se han adherido a estas políticas y en Chile ya se encuentra en trámite un proyecto de Ley de Educación Emocional. Por lo tanto, la preocupación por la dimensión emocional (y/o socioemocional) en la educación, actualmente se encuentra generalizada:

(...) los jóvenes que tienen una base social pueden prosperar mejor en un marco laboral sumamente dinámico $\mathrm{y}$ determinado por las habilidades si perseveran y trabajan mucho (...) Al controlar las emociones y adaptarse al cambio, también pueden estar más preparados para capear las tormentas de la vida, como la pérdida del empleo, la desintegración familiar, la internación y la victimización (OCDE, 2016, p. 26).

Por lo anterior, han proliferado estudios sobre emociones en el campo educativo y psicológico. En una investigación realizada por Toledo y Bonhomme (2019), en la que se revisaron 25 artículos de investigadores/ as chilenos/as que versan sobre el desarrollo socioemocional vinculado a la educación, se encontró que los conceptos sobre emociones, en general, comparten tres aspectos centrales: (a) la dominancia del paradigma cognitivo y el predominio del modelo de la inteligencia emocional, que entiende las emociones como una habilidad y/o competencia al servicio de la adaptabilidad; (b) la incorporación de los objetivos de la salud mental en la escuela para legitimar la inserción de las emociones en el campo de la educación; y (c) la reconfiguración de los roles del profesor y el estudiante en la escuela, incorporando las teorías del apego a la relación profesor alumno, atribuyéndole una función terapéutica/reparadora a dicha relación. El presente artículo propone una revisión crítica desde el alcance de estos hallazgos, desarrollando la problemática educacional derivada de ellos, mediante un marco teórico basado en las conceptualizaciones que subyacen a las ideas de emociones dominantes en el campo educativo. Luego se presenta una investigación teórica basada íntegramente en la obra de Lev Vygotsky con el objeto de sistematizar una teoría de las emociones desde dicho autor.

Como objetivo del artículo, se pretende dar una respuesta a la problemática elaborada teniendo en cuenta el concepto de emociones que se desprende de la obra de Vygotsky, como alternativa a las teorías existentes, de modo que las escuelas tengan la posibilidad de promover un desarrollo socioafectivo, entendiendo la realidad situada de los estudiantes. La tesis que guía este propósito es que las emociones se involucran en procesos de desarrollo similares y mediante los mismos mecanismos que el resto de los procesos psíquicos superiores, y son fundamentales las nociones de monismo entre las funciones y sistemas psicológicos, de imaginación y la de transformación de las emociones.

\section{Cognición e inteligencia emocional}

Desde una mirada crítica, el predominio de una perspectiva cognitiva asociada al estudio de las emociones y su desarrollo impide, de manera categórica, una compleja conceptualización respecto de estas y la dimensión emocional de la escuela. Según Sisto (2006), desde el paradigma de las ciencias cognitivas, el sujeto es entendido como un sistema de procesamiento de datos que consiste en ordenar y categorizar la realidad, por lo que el sujeto y el entorno funcionarían independientemente entre sí. Siguiendo a Palmero (2003), una de las propuestas de esta perspectiva es la adaptación de los sujetos a la realidad, enten- 
dida como una mejor representación de esta a través de una implementación adecuada de las reglas de procesamiento, lo que supone realizar una evaluación y valoración correcta del entorno.

Las ciencias cognitivas, que solían dejar fuera de su campo de estudio otros procesos psicológicos vinculados a la experiencia subjetiva del individuo como las emociones (Sisto, 2006), con la introducción de la noción de Inteligencia Emocional (IE) propuesta por Mayer y Salovey (1990) logran superar las limitaciones de su campo de estudio, capitalizando -gracias sobre todo a la popularización del término a manos de Goleman (2010)la dimensión emocional de los procesos humanos en diversas áreas de la actividad psicosocial, tales como son las empresas, la escuela, la formación militar, entre otras (véase Illouz, 2007, 2010). El modelo de la IE es definido como la habilidad para evaluar las propias emociones y las de otros para discriminarlas “...y usar esa información para guiar el pensamiento y la acción” (Mayer y Salovey, 1990, p. 189). De esta manera, las emociones se introducen en la medida que sirven a la cognición como un elemento que se desglosa en una serie de habilidades orientadas a la consecución de objetivos en la vida cotidiana o la educación.

Para ciertos investigadores chilenos, la IE sería: “...una inteligencia genuina basada en el uso adaptativo de las emociones, de manera que el individuo pudiese solucionar problemas y adaptarse de forma eficaz al medio que le rodea" (Antezana-Saguez, Avendaño-Robledo, Cuadra-Peralta, Fuentes-Soto y Veloso-Besio, 2013, p. 356). De esta manera las emociones son concebidas, por un lado, en cuanto a su función adaptativa y, por otro, como entidades oscuras que deben ser controladas y evaluadas. Desde este punto de vista, es posible hablar de emociones buenas y malas, emociones correctas e incorrectas; apropiadas e inapropiadas, etcétera.

Al basarse en el modelo representacionista, esta teoría no cuestiona el sentido propio de la emoción, ni tampoco los aspectos políticos implicados en las condiciones de verdad para la representación adaptativa. Por otro lado, esta teoría obvia la posibilidad de transformar las emociones y el carácter productivo de estas para la transformación de la experiencia. Asimismo, desestima la posibilidad de que se pueden aprender e inventar emociones, por ello lo que se desestima también es el potencial transformador y creativo de la educación escolar.

\section{Teorías del apego y salud mental: la escuela del carenciado}

Es posible apreciar que las teorías e investigaciones chilenas revisadas por Toledo y Bonhomme (2019) estratifican el desarrollo emocional de acuerdo a cómo el entorno de origen nutre las habilidades emocionales del estudiante, y esto se hace sobre todo recurriendo a teorías psicológicas del vínculo y el apego.

La teoría del apego tiene su origen en el campo de la psicología del desarrollo y fue elaborada por John Bowlby. Cabe señalar que si bien esta teoría surge a partir de las observaciones que el mismo Bowlby realiza con niños institucionalizados (Moneta, 2014), lo cierto es que aporta orientaciones normativas respecto de cómo debe comportarse la figura significativa del niño -usualmente la madrepara prevenir en él un aparato psíquico disfuncional. Desde estas prespectivas, la relación temprana madre-hijo es considerada como el periodo y espacio crucial para la configuración de los modos correctos de sentir y de actuar (Burman, 2008) y asimismo, de representar correctamente la realidad para una adaptación óptima. Los problemas emocionales del niño pasan a ser parte de su dimensión patológica así como también de la de su entorno inmediato que es considerado peligroso (Burman, 2008). Las habilidades desglosadas de la inteligencia emocional del niño entonces, resultan de gran valor para la agenda política de un Estado que requiere sujetos autónomos y participes 
activos de la vida social (Burman, 2008), pero en última instancia sería el entorno inmediato -y para las teorías y estudios revisados, más precisamente el vínculo de apego madrehijo- el que acarrea responsabilidad sobre los efectos indeseados de la sociedad (Burman, 2008). El estudiante es visto como carenciado, pero al ser esto producto de las carencias del ambiente, la patología es concebida como una condición, en su sentido individual (Abramowski, 2010; Ascorra, Arias y Graff, 2003; Burman, 2008). No solo el estudiante es un sujeto clínico, sino también el ambiente inmediato que lo rodea es visto como patológico. Desde las teorías de apego, lo que acontece de los vínculos primarios determina lo que el sujeto es y será (Burman, 2008) y las carencias de estas habilidades son tanto del sujeto como de su entorno.

Gran parte de las publicaciones existentes hacen referencia a esta teoría para explicar la relación profesor-estudiante. Así, los autores proponen que al ser el profesor una figura significativa para los alumnos, actualiza un importante rol de apego:

El apego es dinámico (se enriquece, remodela y está sujeto a la experiencia de la persona) y dura toda la vida, por eso cuando los niños van ampliando su mundo fuera del hogar encuentran otras figuras de apego, como puede ser el profesor (Toro y Berger, 2012, p. 19).

El lenguaje clínico, desde el que derivan las teorías del apego, instalaría no solo una manera particular de entender los problemas educacionales vinculándolos a la inestabilidad del entorno o tipos de apego particulares, sino que también orientaría su forma de intervención: "a problemas diagnosticados como emocionales no pueden no corresponderle soluciones emocionales" (Abramowski, 2017 , p. 256). Quienes se instalan como los conocedores de las emociones, aquellos que saben cómo escucharlas y cómo sentirlas, serán los especialistas de las ciencias psy y lo que deviene es un ethos terapéutico en el que los problemas se conciben tautológicamente gracias a su determinismo emocional (Abra- mowski, 2017; Illouz, 2010). La tautología que propone el ethos terapéutico instala a la emoción como el origen de la carencia (Illouz, 2010), tal como dice Ahmed (2015): "el lenguaje cotidiano de la emoción se basa en la presunción de interioridad" (p.31). Se deifica al psicólogo como aquel que puede ver al interior de la psiquis o quien orienta al sujeto para que pueda buscar en su interior el origen de la emoción, identificarla, nombrarla, escucharla y explorarla (Ahmed, 2015; Illouz, 2010). En el fondo, no cualquiera puede experimentar su emoción (Illouz, 2007).

\section{Sobre los roles educativos: ¿Qué es del profe y qué es de la escuela?}

Ascorra y equipo (2003) en un estudio llevado a cabo en una escuela ubicada en un sector que ha sido estigmatizado como pobre y marginal, dentro de la comuna de Villa Alemana (Chile, quinta región), muestran cómo los agentes educativos y la institución en sí misma transforman su rol de modo que este se edifique en torno a una identidad del alumno configurada por sus carencias:

(...) la percepción que tiene la profesora acerca de su rol, corresponde a un rol que está abocado a suplir carencias del alumno, principalmente, en el ámbito familiar. En este sentido, su rol representa un mundo maternal, asumiendo diversas responsabilidades extraescolares, en perjuicio de sus funciones pedagógicas (Ascorra et al., 2003, p. 132).

No solo los profesores se identifican con un rol materno, orientado a suplir las carencias afectivas de los estudiantes, sino también la escuela misma empieza a ser concebida como un hogar de acogida (Ascorra et al., 2003, p. 133). El rol de la escuela no se orienta según su función educativa, sino según un "rol institucional que busca compensar las necesidades sociales más urgentes de la población beneficiada" (Ascorra et al., 2003, p. 133). Es así que la escuela pierde su función transformadora respecto a las problemáticas sociales y se convierte, tal como se dijo, en un espacio 
de acogida (Ascorra et al., 2003) orientada a contener las emociones derivadas de injusticias sociales y estigmatizaciones de las que el Estado debiese ser responsable (Burman, 2008).

Bajo estos supuestos, las escuelas ahora deben hacerse cargo del famoso ethos terapéutico y convertirse en verdaderas zonas de contención afectiva (Ascorra et al., 2003). Así como el sujeto debe saber controlar y gestionar sus emociones, la escuela debe hacerse cargo de las dimensiones afectivas que le atañen, y además debe procurar el desarrollo y bienestar emocional y socioafectivo de los estudiantes y los partícipes en su interior.

En cuanto a lo expuesto, la forma en que son entendidas y abordadas las emociones en la escuela está en gran medida permeada por las teorías que se encuentran a la base de su conceptualización. En síntesis, tal cual son entendidas las emociones reproducen en la escuela un discurso representacionista y cognitivo, como también una estratificación de las competencias emocionales de los estudiantes de acuerdo a su sector y familia de origen, beneficiando la capitalización exitosa de las emociones por parte de los sectores dominantes de la sociedad. Esto, en última instancia, implica el impedimento de los establecimientos educativos de ejercer su rol transformador, terminando por reproducir la desigualdad social ya existente.

Por todo lo anterior, hace falta una teoría de las emociones que permita transformar la experiencia educativa de modo coherente con los principios constructivistas, para que las escuelas tengan la posibilidad de promover un desarrollo socioafectivo en estrecha relación al desarrollo de conceptos, pero entendiendo la realidad situada histórico-cultural de los estudiantes.

Como se dijo, el presente artículo propone el concepto de las emociones que se desprende de la obra de Vygotsky como una alternativa a las teorías existentes y que cumple con las características mencionadas. Para ello se requiere elaborar una (re)construcción del concepto de emociones presente en la obra de este autor, en coherencia con su sólida teoría de los procesos psicológicos.

En primer lugar, se analizará la perspectiva vygotskyana como una perspectiva monista respecto al intelecto y la emoción. Vygotsky es crítico respecto a la tradición cartesiana que da pie a teorías centrípetas (periféricas y mecanicistas) y a teorías centrífugas (centralistas y espiritualistas) de las emociones. Estas perspectivas suponen una visión dualista entre cuerpo y mente. Luego se discutirá la relevancia del lenguaje como herramienta fundamental en el desarrollo ontogénico histórico-cultural humano y la configuración de sistemas psicológicos superiores. Se profundizará también los conceptos de sistemas psicológicos y el de transformación para entender el desarrollo de los diversos procesos psicológicos, incluyendo las emociones.

\section{El monismo vygotskyano}

Vygotsky (1930/2014) se apoya en la filosofía spinoziana para debatir el dualismo subyacente a las teorías mecanicistas y espiritualistas de las emociones en aquella época. Si bien no concibe el intelecto y las emociones como una misma función psicológica, no se puede explicar ni el intelecto ni las emociones de forma pura dentro de la vida y la experiencia de los sujetos.

Para Vygotsky (1930/2014), Spinoza poseía una actitud genética correcta debido a que "(...) afirmaba que el hombre tiene poder sobre los afectos, que la razón puede alterar el orden y las conexiones de las emociones y hacer que concuerden con el orden y las conexiones dados en la razón" (p. 87). De acuerdo con esto, Vygotsky (1933/2017) en su obra "Teoría de las emociones. Estudio histórico-psicológico", desarrolla una profunda revisión acerca de las teorías clásicas y contemporáneas sobre de las emociones. Analiza la continuidad de las concepciones filosóficas epistémicas que se encuentran a la base del ejercicio científico de su época. Más precisa- 
mente, intenta realizar una crítica a la continuación de la concepción dual cartesiana entre las nociones mecanicistas y espirituales que se encuentran en la médula de las concepciones teóricas de entonces:

Para nosotros, lo único que importa, al acabar el examen de la suerte de la teoría cartesiana de las pasiones en la psicología contemporánea, es mostrar que dicha teoría estaba polarizada por los principios contradictorios que encerraba y que se ha encarnado en las posiciones extremas, mecanicistas y espirituales, de la psicología contemporánea (Vygotsky, 1933/2017, p. 255).

Siendo más preciso, Vygotsky (1930/2014) expuso la idea categórica de que las emociones entran en relación tanto con otras funciones específicas (como el pensamiento, por ejemplo) así como también con otros sistemas aún más complejos. Dichas relaciones permiten desarrollar un monismo funcional en el que las emociones no pueden operar de manera aislada. Vygotsky (1930/2014) lo explica de la siguiente forma:

(...) nuestros afectos actúan en un complicado sistema con nuestros conceptos y quien no sepa que los celos de una persona relacionada con los conceptos mahometanos de la fidelidad de la mujer son diferentes de los de otra relacionada con un sistema de conceptos opuestos sobre lo mismo, no comprende que ese sentimiento es histórico, que de hecho se altera en medios ideológicos y psicológicos distintos, a pesar de que en él queda indudablemente cierto radical biológico, en virtud del cual surge esta emoción. (Vygotsky, 1930/2014, p. 87)

Ahora bien, la forma mediante la cual los afectos y el intelecto se ponen en relación debe ser analizada de acuerdo con cómo Vygotsky entiende el desarrollo sociohistórico de los seres humanos: “(...) el desarrollo histórico de los afectos o las emociones consiste fundamentalmente en que se alteran las conexiones iniciales en que se han producido y surgen un nuevo orden y nuevas conexiones" (Vygotsky, 1934/2014, p. 87). Pues desde el punto de vista de Vygotsky, el desarrollo humano ontogenético deriva de las conexiones interfuncionales entre las distintas funciones psicológicas que son compartidas con otros homínidos.

\section{Lenguaje intelectual o Pensamiento verbal: Piedra angular del psiquismo humano}

En el capítulo 4 de "Pensamiento y Lenguaje", Vygotsky (1934/2014) argumenta que el pensamiento y el lenguaje serían dos funciones psicológicas distintas que siguen líneas de desarrollo diferentes, pero que en determinado momento convergen transformando así el propio pensamiento, siendo este momento el punto crucial para la emergencia y el desarrollo del pensamiento verbal puramente humano. Para Vygotsky, tanto el pensamiento como el lenguaje por sí solos no son funciones psicológicas propiamente humanas; por el contrario, argumenta apoyándose en variados ejemplos de experimentos anteriores que dichas funciones ya están presentes en otros animales, sobre todo en otros homínidos (Vygotsky, 1934/2014). La particularidad del humano es que, en cierto momento de su desarrollo ontogenético, estas funciones logran operar de manera indisoluble, emergiendo una especie particular de lenguaje (intelectual) o pensamiento (verbal). Desde entonces, este tipo pensamiento pasará a ser herramienta organizadora de la actividad psíquica del sujeto, capaz de posibilitar diversas conexiones funcionales (Vygotsky, 1934/2014). Ante esto, corresponde enfatizar que las funciones psicológicas no son las que se desarrollan unitaria e intrafuncionalmente, sino que lo que varía y se desarrolla son los "nexos interfuncionales, de la estructura interfuncional" (Vygotsky, 1930/2014, p. 73). Surgen nuevos agrupamientos que responden al funcionamiento del sujeto -y que adquieren sentido- en el transcurso de su vida histórico-cultural. Por lo tanto, como dice Vygotsky:

(...) el pensamiento verbal no constituye una forma natural de comportamiento, sino una 
forma histórico social, que debido a ello se distingue en lo fundamental por toda una serie de propiedades y regularidades específicas, que no pueden encontrarse en las formas naturales del pensamiento (1934/2014, p. 117).

Lo que facilita el tipo de pensamiento verbal, es el uso de signos socioculturales como herramienta, de modo que este nexo interfuncional entre el pensamiento y lenguaje sirve de base para la articulación de otros nuevos nexos entre funciones psicológicas (Vygotsky, 1930/2014).

En la teoría vygotskyana, el signo como herramienta es de fundamental importancia, pues para Vygotsky "[e]l hombre, a su vez, posee un sistema de actos que limita las formas de su conducta, pero amplía el radio de sus acciones, a diferencia de otros seres, gracias a la ayuda de herramientas" (1931/2012,p. 331). En este caso, el signo sería una herramienta orientada a ampliar el radio de las acciones del sujeto, en principio externo al niño, y partícipe de su vida social: “[e]l signo, al principio es siempre un medio de relación social, un medio de influencia sobre los demás y tan solo después se transforma en medio de influencia sobre sí mismo" (Vygotsky, 1931/2012, p. 146). Gracias a la necesidad por la utilización del signo como herramienta -necesidad que surge de la mediación externa y social-, es que el sujeto transita a la confluencia entre pensamiento y lenguaje.

Para Vygotsky un proceso externo es un proceso social $(1931 / 2012,1930 / 2017)$ y continuando su argumento, la historia del desarrollo cultural del hombre no coincide ni es producto de la biología ni de la evolución filogenética. La historia de estas confluencias entre los procesos psicológicos es la historia del dominio de la conducta del hombre mediante el uso de signos que le permiten transformarse a sí mismo y la naturaleza. En este sentido, cabe hablar del trazado cultural-histórico que carga el desarrollo humano:

Justamente la cultura es un producto de la vida social y de la actividad social del ser humano (...) Podríamos señalar, además, que el signo, que se halla fuera del organismo, al igual que la herramienta, está separado de la personalidad y sirve en su esencia al órgano social o al medio social (Vygotsky, 1931/2012, p. 151).

De acuerdo con esto, las formas superiores de comportamiento son el resultado de la historia cultural del niño o niña mediante el ejercicio de la actividad mediadora, que refiere al uso de signos, que en primera instancia le son externos (Vygotsky, 1931/2012, 1930/2017). La génesis del signo tiene lugar en el gesto indicativo, al encuentro con el objeto, concibiéndose como un sistema primario de signos (Vygotsky, 1931/2012). En "La prehistoria del desarrollo del lenguaje escrito" (1931/2012), Vygotsky analiza la génesis e historia del lenguaje escrito desde el gesto al signo y la emancipación de este -función simbólicamediante el juego; y luego el dibujo como lenguaje gráfico que antecede a la capacidad de dibujar palabras -lenguaje escrito (1931/2012). La escritura, que generalmente se enseña en la escuela, tiene su génesis en las transformaciones culturales del uso del signo, en las que se establecen saltos cualitativos en muchas ocasiones conflictivos- entre un estado anterior y otro (Vygotsky, 1931/2012):

(...) cuando el niño se adentra a la cultura, no solo toma algo de ella, no solo asimila y se enriquece con lo que está fuera de él, sino que la propia cultura reelabora en profundidad la composición natural de su conducta y da una orientación completamente nueva a todo el curso de su desarrollo (Vygotsky, 1931/2012, p. 305).

Desde aquí el curso de la actividad práctica del sujeto es "transformada" gracias a la adquisición de una nueva herramienta capaz de organizar la conducta y prácticamente todos los componentes de su vida psíquica. Vygotsky estudia el significado de la palabra como la unidad que responde a las propiedades inherentes del pensamiento verbal (1934/2014):

Hemos encontrado esta unidad, que refleja la unión del pensamiento y el lenguaje, en la forma más simple, en el significado de la 
palabra. El significado de la palabra, como hemos intentado explicar anteriormente, es la unidad de ambos procesos, que no admite más descomposición y acerca de la cual no se puede decir qué representa: un fenómeno del lenguaje o del pensamiento (p. 289).

El significado de la palabra, entonces, corresponde a un fenómeno verbal e intelectual a la vez (Vygotsky, 1934/2014), cuya relevancia es que en ella el uso de signos se pone al servicio del sujeto en la realización de tareas específicas. La unidad arquitectónica de la estructura psíquica humana corresponde al significado de la palabra, pues en él encuentran unidad el pensamiento y el lenguaje (Vygotsky, 1934/2014). La conducta del sujeto puede ser controlada por este gracias al uso simbólico de la palabra adquiriendo un dominio particular de su actividad sociocultural (Vygotsky, 1934/2014, 1930/2014). El lenguaje se encuentra entonces con el pensamiento -que operaba independientemente con fines netamente prácticos que en nada se distingue al de otros homínidos- en su implicancia sociohistórica, justamente en el signo, funcionando como entidad organizadora de toda la actividad humana (Vygotsky, 1930/2017): "Nuestro análisis concede a la actividad simbólica una específica función organizadora que se introduce en el proceso del uso de instrumentos y produce nuevas formas de comportamiento" (p. 47). La actividad simbólica, entonces, "engendra por vez primera formas humanas propias de utilización de instrumentos" (Vygotsky, 1930/2017, p. 26).

El pensamiento verbal -que tiene como unidad el significado de la palabra- incorpora en la práctica intelectual del niño o niña una dimensión temporal entre la percepción y la conducta. En fases tempranas del desarrollo los procesos sensorial y motriz funcionan de manera idéntica y próxima, como un puro sistema (sensorio-motriz). La particularidad, según Vygotsky (1930/2014) radica en que "los procesos motores y sensoriales, interpretados en el plano psicológico, adquieren una relativa independencia mutua, relativa en el sentido de que ya no existe la unidad, la relación directa, propia del primer nivel de desarrollo" (p. 74). A simple vista esto parece una contradicción-sobre todo si se le compara con la relación entre pensamiento y lenguaje. No obstante, esto se relaciona con que la función sensorio-motriz funcionaría como un sistema primario entre dos funciones estrechamente relacionadas. Lo que ocurre luego no es una separación, ni tampoco una pérdida de estas funciones, sino una transformación en que cada una de estas, al entrar en relación con nuevas funciones, forman parte de un sistema aún más complejo, por lo que se establece no un corte, sino un cúmulo de nuevas posibilidades entre ambas.

\section{Los sistemas psicológicos y el desarrollo como transformación}

En "Sobre los sistemas psicológicos", Vygotsky (1930/2014) analiza las relaciones interfuncionales entre funciones psicológicas, capaces de formar sistemas psicológicos. El sistema psicológico se entiende como un monismo funcional entre distintas funciones psicológicas como lo son la memoria, atención, pensamiento, lenguaje, percepción, emociones, motricidad, entre otras. De esta manera, las diferentes operaciones incorporan las otras funciones en mayor o menor grado, impidiendo aislar cada función a su componente orgánico. Uno de los ejemplos que Vygotsky en dicho texto analiza para mostrar el monismo funcional en el que actúan las funciones psicológicas, es justamente el de la percepción:

(...) El conocimiento del objeto resulta simultáneo a la percepción del mismo (...) Una vez aislada de la motricidad la percepción no continúa desarrollándose intrafuncionalmente, sino que el desarrollo se da precisamente debido a que la percepción establece nuevas relaciones con otras funciones, entra en complicadas combinaciones con nuevas funciones y comienza a actuar conjuntamente 
con ellas como un sistema nuevo, que resulta bastante difícil de descomponer y cuya desintegración tan solo puede observarse en patología (1930/2014, p. 76).

Así, la constitución de sistemas psicológicos no implica una mera colaboración entre funciones, sino la capacidad de transformación mutua. El hecho de que uno piense verbalmente no solo implica una transformación tanto del lenguaje como del pensamiento como funciones, $\mathrm{y}$, como se expresa en la cita anterior, prácticamente una forma superior e irreversible de pensamiento verbal (Vygotsky, 1934/2014, 1930/2014). A su vez, esta nueva forma de pensamiento es capaz de transformar y ser transformada por otras funciones de modo que los sistemas psicológicos son producto de estas transformaciones.

Ahora bien, la unidad estructural que permite la articulación entre funciones es el pensamiento verbal a través del uso del signo social externo. El motor de aquella transformación se encuentra en la actividad social y el encuentro con otros quienes incitan y dan valor al uso del signo (Vygotsky, 1934/2014). Y el fin último de estas transformaciones se relaciona con la idea de aumentar la capacidad de acción del sujeto orientada al dominio de la realidad y los objetos externos (Vygotsky, 1931/2012).

Los procesos psíquicos superiores transitan procesos evolutivos similares a las transformaciones que le anteceden, pero que solo pueden tener lugar en la vida sociohistórica. En este sentido, los procesos de dominio como procesos psíquicos superiores- también se desarrollan, pero estos son análogos y hasta se derivan de la capacidad de dominio que el hombre tiene sobre la naturaleza:

Queda admitir que nuestro dominio sobre los procesos propios del comportamiento se construye en esencial, de la misma manera que nuestro dominio sobre los procesos de la naturaleza, ya que el hombre que vive en sociedad está siempre sujeto a la influencia de otras personas (Vygotsky, 1931/2012, p. 290).

El proceso de desarrollo de estos dominios psíquicos se explica por las transformaciones de las funciones psíquicas al hacerse parte orgánica de un cuerpo sistémico en que participan. Y como tal, la capacidad de transformar nuestra voluntad es, en realidad, la capacidad que tienen los objetos (externos) de transformarla. Vygotsky lo expresa de este modo:

El hombre, utilizando el poder de las cosas o estímulos sobre su conducta, pasa a dominar por mediación de ellos (...) su propio comportamiento. Dicho de otro modo: la inmensa peculiaridad de la voluntad consiste en que el hombre no tiene poder sobre su propia conducta, a excepción del poder que tienen las cosas sobre ella (1931/2012, p. 292).

Ahora bien, el nexo entre los mecanismos funcionales no solo aumenta la capacidad de nuestra conducta gracias a que se origina un sistema de relaciones que nos permite organizar y planificar nuestro comportamiento. Según la teoría vygotskyana, este sistema de funcionamiento no responde a lógicas de niveles de conciencia o diferencias en los tipos de pensamiento que nos permiten orientar la acción. Un ejemplo de esto es la crítica que desarrolla Vygotsky (1932/2014b) a la clásica distinción entre pensamiento autista y pensamiento lógico.

La tradición clásica del psicoanálisis propone la existencia de una forma de producción imaginaria en la que el niño o niña crea imágenes de acuerdo con los principios de su pensamiento guiados primordialmente por el placer; un tipo de pensamiento caracterizado por la supremacía del mundo interno y la satisfacción de placeres intrínsecos, denominado por Piaget como "pensamiento autista" (Vygotsky, 1932/2014a). Únicamente gracias a los procesos de adquisición del lenguaje mediante medios socializadores, el niño puede transitar hacia un formato de pensamiento orientado a la realidad (mundo exterior) y los procesos lógicos compartidos socialmente: Pensamiento lógico (Vygotsky, 1932/2014b). Vygotsky critica que la génesis de la imaginación esté relacionada con procesos irracionales, prelingüísticos, incomunicables y orientados por un placer innato. Para Vygotsky, este 
tipo de perspectivas “...considera al hombre como un ser que al principio no se desarrolla socialmente, sino a quien la actividad social se le agrega como algo externo, secundario" (1932/2014b, p. 430). Por el contrario, Vygotsky entiende que la condición de posibilidad para el surgimiento de la imaginación es el dominio del lenguaje como herramienta y que no existiría algo así como un tipo de pensamiento en función de la satisfacción personal previa al dominio lenguaje (1932/2014b). Según Vygotsky, previo al lenguaje no hay indicios de que la acción del niño se encuentre orientada a la satisfacción del placer ni al agotamiento de descargas energéticas primordiales. Más bien, no hay más que una orientación del niño hacia la satisfacción de formas simples de necesidades vitales que guardan relación con la actividad y conocimiento del mundo real (Vygotsky, 1932/2014b). Entonces, estos tipos de pensamiento -que pueden ser entendidos como niveles temporales en el desarrollo y como agregados intrafuncionales (en que lo cultural se le agrega a un tipo de pensamiento primitivo, instintivo)- deben entenderse como un solo proceso que adquiere cualidad de acuerdo con la funcionalidad del pensamiento en su despliegue sociocultural.

El dominio del lenguaje no deriva de un proceso de socialización que reprime los impulsos primitivos del sujeto, ni que funciona como tamiz del intelecto cultural. Tampoco refiere a que la imaginación sea un sustrato indeseable dominado por deseos y placeres inconscientes que amenazan por refrenar la actividad productiva del hombre. La imaginación, en cambio, constituye para Vygotsky un proceso en el cual las emociones juegan un rol fundamental dentro de un sistema tanto afectivo como intelectual, tan creativo como realista (1932/2014b). Por ello, la imaginación es una forma particular de pensamiento orientado a la vida práctica, que se caracteriza “(...) no por una mejor conexión con el aspecto emocional, no por su grado menor de conciencia, no por un grado menor ni mayor de concreción; esas cualidades se manifiestan también en las distintas etapas del desarrollo del pensamiento" (Vygotsky, 1932/2014b, p. 437). El desarrollo de la imaginación coincide en cierta medida con el desarrollo del pensamiento entendido como la convergencia entre pensamiento y lenguaje:

Al igual que el desarrollo del pensamiento infantil, en el desarrollo de la imaginación, el momento crítico principal coincide con la aparición del lenguaje. La edad escolar es el punto crucial en el desarrollo del pensamiento infantil realista y autista. Dicho de otro modo, vemos que el pensamiento lógico y el pensamiento autista se desarrollan en una excepcional y estrecha relación (1932/2014b, p. 437).

La imaginación funciona como un proceso en que las emociones son transformadas y estas mismas transforman el pensamiento, ampliando el rango espacial en que este $\mathrm{y}$ nuestra capacidad de acción operan.

\section{Emociones}

Vygotsky concuerda con la noción de Spinoza sobre las emociones, “...para quien los afectos son un estado del cuerpo que aumentan o disminuyen la capacidad de actuar del propio cuerpo, favoreciéndola o limitándola y al mismo tiempo las ideas de estos estados" (1930/2017, p. 112). No obstante, estos estados del cuerpo son mucho más que simples estados. La transformación de dichos estados que aumentan la capacidad de acción no tiene tanto que ver con la capacidad del sujeto para controlar, organizar y planificar su conducta. Cabría tener en cuenta lo que en "Teoría de las Emociones" Vygotsky intenta destacar respecto a estos estados o cambios corporales relacionados con los afectos:

El quid de la cuestión consiste, por consiguiente, no en la existencia de estos cambios durante las emociones, sino en la actitud de estos cambios corporales hacia el contenido psíquico y la estructura de las emociones, por un lado, y a su significado funcional, por otro (1930/2017, p. 113). 
No es lo relevante la existencia o no de esos estados, sino de la relación que tienen las emociones o afectos dentro del sistema psicológico particular, así como también el sentido que dicha función imprime a la actividad humana. Cuando Vygotsky habla de aumentar la capacidad de acción, seguramente no solo refiere a las posibilidades que se abren dentro del espacio entre lo sensoriomotriz, como control de la conducta ni tampoco una mera posibilidad de planificar la acción. Argumentaré usando a Vygotsky que la capacidad de aumentar la acción permite el funcionamiento de un complejo sistema no solo relacionado con el dominio de la conducta - pese a que esto pueda ser considerado su psicogénesis-, sino que también emerge la posibilidad de planear, imaginar, pensar en mundos posibles, incluso soñar despiertos las posibilidades del mundo o lo que el mundo -o nuestro mundo- podría haber sido.

Ahora bien, hemos visto que las emociones son transformadas por el intelecto mediante el desarrollo del dominio de la conducta, la imaginación, la voluntad y el sentido. Las emociones no son simples descargas, sino que en todo momento son producidas, creadas, transformadas, mediatizadas, etcétera, sin que estas pierdan su fuerte radical biológico material. Gracias al enriquecimiento y los cambios en los usos del lenguaje en el desarrollo sociocultural, estas cobran nuevas relaciones para su transformación: “(...) La reacción emocional es el resultado singular de una estructura concreta del proceso psíquico" (Vygotsky, 1932/2014b, p. 419). En este sentido, la transformación íntegra de la estructura del proceso psíquico determina el curso del desarrollo de la reacción emocional, transformándola también.

Esta tesis propone que las emociones se desarrollan, se transforman, se aprenden, gracias y mediante los mismos mecanismos que Vygotsky describe para el resto de las funciones psíquicas superiores. Por ende, cobra relevancia el papel que tiene el sistema escolar en la promoción de nuevas formas de pensamiento, pues en la escuela es donde el niño:

(...) puede pensar minuciosamente algo en forma imaginada, antes de llevarlo a cabo. Eso constituye indudablemente la base de que precisamente durante la edad escolar se establezcan las formas primarias de la capacidad de soñar en el sentido propio de la palabra, es decir, la posibilidad y la facultad de entregarse más o menos conscientemente a determinadas lucubraciones mentales, independientemente de la función relacionada con el pensamiento realista (Vygotsky, 1932/2014b, p. 433).

El proceso mediante el cual se produce el desarrollo cultural no es un proceso lineal, sino un proceso interrumpido, lleno de contradicciones y conflictos en el que se yuxtaponen, oponen, imbrican los procesos orgánicos y cultuales (véase Vygotsky, 1931/2012). Esto último tiene efectos e implicaciones concretas para el sistema educativo, pues “(...) [e]n efecto, para la educación no es lo mismo que el niño pase en línea recta del balbuceo a la palabra o de la percepción de la figura numérica al sistema decimal" (Vygotsky, 1931/2012, p. 306). En el aprendizaje escolar, tal como se imbrican procesos intelectuales complejos con otros más o menos sencillos, así también es posible transformar las emociones, como también producir emociones gracias al uso de dimensiones imaginarias complejas dotadas por el sistema escolar.

Las formas de enseñanza requieren ser pensadas como técnicas sociales de afección (así como Vygotsky, 1925/2006, concibe a la obra de arte) que afectan y que transforman las formas de pensar, imaginar y emocionarse, arraigadas a una situación social específica de aprendizaje y desarrollo histórico, y culturalmente diseñados para tal efecto. El desarrollo íntegro de cada función y sistema psicológico no es independiente de cada uno, sino que el desarrollo de los procesos psíquicos superiores refiere al desarrollo de un todo orgánico que sufre nuevas transformaciones, mutaciones y conjunciones de manera dinámica, pero no predeterminada ni de forma arbitraria, 
sino situada en procesos históricoculturales de afección social.

\section{Discusión y conclusiones}

Una primera lectura de la teoría vygotskyana corre el riesgo de entender la relación del pensamiento - como pensamiento verbal-y las emociones desde la misma lógica del control de la conducta (véase Vygotsky, 1931/2012, $1932 / 2014 c, 1930 / 2017)$ y es que el lenguaje y pensamiento se vinculan con las emociones de un modo que efectivamente se encuentra estrechamente ligado con la pregunta acerca del desarrollo de la voluntad en Vygotsky (véase 1931/2012, 1932/2014c). De este modo, la teoría vygotskyana podría seguir la misma lógica representacionalista de las ciencias cognitivas y de regulación y gestión emocional de la inteligencia emocional. Sin embargo, al haber analizado los textos orientados al resto de los procesos superiores, vemos que Vygotsky (véase 1931/2012, 1934/2014), entiende los procesos de desarrollo más como transformaciones que como un desarrollo progresivo y lineal en cuanto a sus cualidades.

En segundo lugar, argumentar que las emociones se transforman gracias a la vía del desarrollo histórico, cultural y social en la ontogénesis $-y$ que estas transformaciones refieren más a saltos, o rupturas, que pueden ser vistas como conflictivas respecto del desarrollo orgánico del sujeto (véase Vygotsky, 1931/2012)-, permite entender la educación escolar como una vía transformadora productiva, que no se paraliza ante los problemas que el niño porta desde su entorno inmediato, ni que orienta el foco hacia sus déficits y condiciones. Más bien permite pensar la educación como un sistema que "(...) analice positivamente su personalidad [del estudiante] y la posibilidad de crear vías de desarrollo colaterales, de rodeo" (Vygotsky, 1931/2012, p. 313). Si bien en el contexto del fragmento citado Vygotsky refiere a niños y niñas con algún tipo de déficit orgánico, esto último no constituye el quid de la cuestión, pues el foco está en que los medios culturales de afección presumen ciertas condiciones, ya sean biológicas o sociales de los cuerpos -como puede ser lo que esperamos del vínculo temprano o la educación de la casa-, pero que en muchos casos no tienen ni deben tener precisamente consonancia ni armonía para la concreción de las transformaciones que el medio históricosocial promueve desarrollar. Esto último se vincula con la concepción de Situación social del desarrollo, rescatada por González-Rey (2000) para quien el concepto de vivencia en Vygotsky resulta fundamental para entender el sentido que tienen las transformaciones de los sistemas psicológicos en la situación contextual del aprendizaje presente sobre todo en la tarea como ejercicio de afección.

Las teorías de apego, como se ha visto, se basan en la psicogénesis de las competencias socio-emocionales en el desarrollo temprano, en general sustentadas en la continuidad ontogénica armónica del desarrollo filogenético del individuo puesta en la relación y el vínculo madre-hijo, naturalizando el rol irreversible que tiene la madre $-\mathrm{o}$ el cuidador inmediato- en el futuro desarrollo del sujeto (véase Burman, 2008). Ante esto, se instala un cierto determinismo, que muchas veces justifica que la escuela descanse en labores de contención afectiva (Ascorra et al., 2003) o potenciando la estigmatización social al individualizar el problema como déficit (Abramowski, 2010). En el mejor de los casos, se le confiere al docente, o al cuidador de turno, reactualizar el rol de apego, cumpliendo una función terapéutica (Berger y Toro, 2012). Como respuesta, Vygotsky permite relativizar tanto el rol de los cuidadores ante el desarrollo del niño, desnaturalizando este proceso y situándolo en el plano del desarrollo histórico-cultural. Por otro, tanto el proceso de desarrollo ontogenético primario como el desarrollo orgánico son transformados y conflictuados en el encuentro con otros medios superiores de transformación y desarrollo de procesos psíquicos superiores. Por ende, la escuela no 
requiere ser pensada en cuanto a su rol de continuidad de los procesos tempranos, así como tampoco requiere asumir las competencias sociales provenientes del hogar, las que en última instancia el Estado debiera -si es así- hacerse responsable. Tampoco es rol de la escuela compensar dichas carencias emocionales, sino pensar en la "posibilidad de crear vías laterales de desarrollo" y que "(...) [a]llí donde el desarrollo orgánico resulta imposible, hay infinitas posibilidades para el desarrollo cultural" (Vygotsky, 1931/2012, p. 313) y su transformación. Y esto pues, porque para Vygotsky "la personalidad no representa una entidad intrapsíquica sustancializada (...) sino un sistema complejo integrador de la vida psíquica individual, que participa en el sentido que las experiencias tienen para el sujeto" (González-Rey, 2000, p.146). Por ende, conjeturar que los problemas de desarrollo de los estudiantes derivan de carencias emocionales fundamentales para la constitución de una personalidad específica y substancial, resulta al menos problemático desde un punto de vista vygotskyano, sin siquiera mencionar que subestima el potencial transformador de la escuela y los medios de educación -formales o no- de la sociedad.

Por último, el desarrollo de las emociones no puede ser pensado de forma independiente del desarrollo de otros procesos psíquicos superiores. El análisis que se realizó acerca del monismo vygotskyano y el rol de la imaginación resultan cruciales. Existen autores que proponen la estrecha relación entre la imaginación y la adquisición de conceptos en los procesos argumentativos en contextos de aprendizaje escolar (véase Larraín, 2016). Esto permite extrapolar cómo nuevas experiencias de habla o razonamiento son transformadoras también para las emociones, a la vez que implican afectos en tales formas de razonamiento y habla.

El desarrollo, entendido como la transformación de las emociones, puede ser movilizado mediante procesos de aprendizaje que no requieren hacer referencia a unidades metafísicas internas e individuales del sujeto, ni requiere remitir a estados previos al desarrollo del lenguaje. Cabe más bien pensar en cómo nuestros sistemas culturales de enseñanza - específicamente la escuela- es capaz de transformar las formas de pensar y sentir, afectar y aumentar la capacidad de acción de los estudiantes.

Aún queda como desafío conceptualizar los medios de afección de las emociones. Teórica y empíricamente faltan por analizar las dimensiones estéticas del aprendizaje vinculadas a la transformación de las emociones y las formas particulares de pensamiento que las aulas provocan. Por ende, resultaría relevante estudiar las herramientas efectivas que permiten tanto el desarrollo socioafectivo como la adquisición de conceptos en las aulas, delimitando los roles tanto de los actores educativos, como de la institución misma respecto a sus responsabilidades en la formación de ciudadanos capaces de participar democráticamente.

Finalmente, no da lo mismo el concepto de emociones que se encuentra a la base de las teorías y propuestas educacionales. La psicología es una gran responsable de las confusas implicancias analizadas que impiden el desarrollo adecuado del potencial transformador de la escuela. La teoría de Vygotsky parece entregar mejores luces y abrir camino a estrategias eficaces y responsables, ética y políticamente hablando, al incorporar la dimensión emocional dentro del campo educativo.

\section{Referencias bibliográficas}

\footnotetext{
Abramowski, A. (2010). Maneras de querer. Los afectos docentes en las relaciones pedagógicas. Buenos Aires, Argentina: Paidós.

Abramowski, A. (2017). Legislar los afectos. Apuntes sobre un proyecto de ley de Educación Emocional. En A. Abramowski y S. Canevaro, Pensar los afectos: aproximaciones desde las ciencias sociales y las humanidades (pp. 251-272). Buenos Aires, Argentina: Ediciones UNGS.
} 
Agencia de la Calidad de la Educación. (2014). Factores asociados a resultados simce e indicadores de desarrollo personal y social. http://www.agenciaeducacion.cl/wp-content/uploads/2016/02/Factores asociados Simce_e Indicadores_desarrollo_personal social 2014-.pdf

Agencia de la Calidad de la Educación. (2018). SIMCE. http://www.agenciaeducacion.cl/ evaluaciones/que-es-el-simce/

Ahmed, S. (2015). La política cultural de las emociones. México, D. F.: Universidad Autónoma de México, Instituto de Investigaciones.

Álamos, P., Alcalay, L., Berger, C. y Milicic, N. (2013). Aprendizaje socioemocional en estudiantes de quinto y sexto grado: presentación y evaluación de impacto del programa BASE. Ensaio: Avaliação e Políticas Públicas em Educação, 21(81), 645-666. https://doi. org/10.1590/S0104-40362013000400002

Álamos, P., Marchant, T. y Milicic, N. (2015). Competencias Socioemocionales: Capacitación de Directivos y Docentes y su Impacto en la Autoestima de Alumnos de 3o a 7o Básico. Revista Iberoamericana de Evaluación Educativa, 8(2), 203-218.

Antezana-Saguez, I., Avendaño-Robledo, R., Cuadra-Peralta, A., Fuentes-Soto, L. y Veloso-Besio, C. (2013). Relación entre inteligencia emocional, satisfacción vital, felicidad subjetiva y resiliencia en funcionarios de Educación Especial. Estudios Pedagógicos, (2), 355-366. https://doi.org/10.4067/S071807052013000200022

Ascorra, P., Arias, H. y Graff, C. (2003) La escuela como espacio de contención social y afectiva. Revista Enfoques Educacionales, 5(1), 1-19. .

Burman, E. (2008). Deconstructing developmental psychology (2nd ed). London/New York: Routledge

Carrasco, N., Figueroa, P., Guzman-Gonzalez, M., Trabbuco, C. y Vilca, D. (2016). Estilos de apego y dificultades de regulación en estudiantes universitarios. Psykhe, 25(1), 1-13. https://doi.org/10.7764/psykhe.25.1.706

Casassus, J. (2007). La educación del ser emocional. Santiago de Chile: Cuarto Propio.
Céspedes, A. y Silva, G. (2013). Las emociones van a la escuela, el corazón también aprende. Chile: Calpa y Ábila.

Goleman, D. (2010). La Inteligencia Emocional. Argentina: Vergara.

González-Rey, F. (2000). El lugar de las emociones en la constitución social de lo psíquico: El aporte de Vigotski. Educaçao e Sociedade, ano XXI, 70, 132-148.

Illouz, E. (2007). Intimidades congeladas. Las emociones en el capitalismo. Buenos Aires, Argentina: Katz.

Illouz, E. (2010). La salvación del alma moderna: Terapia, emociones y la cultura de autoayuda. Buenos Aires/Madrid: Katz

Larraín, A. (2016). Argumentation and concept development: the role of imagination. European Journal of Psychology of Education, 32(4), 521-536. https://doi.org/10.1007/ s10212-016-0316-7

Mayer, J. D. y Salovey, P. (1990). Emotional Intelligence. Imagination, Cognition and Personality, 9(3), 185-211. https://doi.org/10.2190/ DUGG-P24E-52WK-6CDG

Moneta, M. (2014). Apego y pérdida: redescubriendo a John Bowlby. Revista Chilena de Pediatría, 85(3), 265-268. http://dx.doi. org/10.4067/S0370-41062014000300001

OCDE. (2016). Habilidades para el progreso social. El poder de las habilidades sociales y emocionales. Montreal: Instituto de Estadísticas de la UNESCO.

Palmero, F. (2003). La emoción desde el modelo cognitvista. Revista electrónica de motivación y emoción, 6, 14-15. http://reme.uji.es/articulos/avillj3022401105/texto.html

Sisto, V. (2006). Acerca de la inexistencia de la ciencia cognitiva. Psicoperspectivas. Individuo y Sociedad, 1(1), 77-102.

Toledo, C. y Bonhomme, A. (2019). Educación y emociones: coordenadas para una teoría vygotskyana de los afectos. Psicologia Escolar e Educacional, 23(4), 0-7. http://dx.doi. org/10.1590/2175-353920190193070

Toro, L. y Berger, C. (2012). Percepción de profesores jefes de quinto y sexto básico de su rol en el aprendizaje socioemocional de los estu- 
diantes. Diálogos Educativos, 12(23), 16-37.

Vygotsky, L. S. (1925/2006). Psicología del Arte. Barcelona, España: Paidós.

Vygotsky, L. S. (1930/2014). Sobre los sistemas psicológicos. En L. S. Vygotsky, Obras Escogidas. Tomo I (pp. 71-93). Madrid, España: Antonio Machado.

Vygotsky, L. S. (1930/2017). El instrumento y el signo en el desarrollo del niño. En L. S. Vygotsky, Obras Escogidas. Tomo VI (pp. 9-100). Madrid, España: Antonio Machado.

Vygotsky, L. S. (1932/2014a). Las emociones y su desarrollo en la edad infantil. En L. S. Vygotsky, Obras Escogidas. Tomo II (pp. 403-424). Madrid, España: Antonio Machado.

Vygotsky, L. S. (1931/2012). Historia del desarrollo de las funciones psíquicas superiores. En L. S. Vygotsky, Obras Escogidas. Tomo III (pp.
10-340). Madrid, España: Antonio Machado.

Vygotsky, L. S. (1932/2014b). La imaginación y su desarrollo en la edad infantil. En L. S. Vygotsky, Obras Escogidas. Tomo II (pp. 423-438). Madrid, España: Antonio Machado.

Vygotsky, L. S. (1932/2014c). El problema de la voluntad y su desarrollo en la edad infantil. En L. S. Vygotsky, Obras Escogidas. Tomo II (pp. 439-449). Madrid, España: Antonio Machado.

Vygotsky, L. S. (1933/2017). Teoría de las emociones. Estudio histórico-psicológico. En L. S. Vygotsky, Obras Escogidas. Tomo VI (pp. 9-100). Madrid, España: Antonio Machado.

Vygotsky, L. S. (1934/2014). Pensamiento y Lenguaje. En L. S. Vygotsky, Obras Escogidas. Tomo II (pp. 9-348). Madrid, España: Antonio Machado.

Recibido: 26 de marzo de 2019 Aceptado: 7 de octubre de 2020 